\title{
On the kinetic stability of magnetic structures in electron drift turbulence
}

\author{
Martin Jucker ${ }^{\text {a) }}$ \\ Ecole Polytechnique Fédérale de Lausanne (EPFL), Centre de Recherches en Physique des Plasmas, \\ Association Euratom-Confédération Suisse, CH-1015 Lausanne, Switzerland \\ Vladimir P. Pavlenko \\ Uppsala University and EURATOM-VR Fusion Association, SE-751 20 Uppsala, Sweden
}

(Received 27 November 2006; accepted 22 January 2007; published online 2 March 2007)

\begin{abstract}
The generation of large-scale magnetic fields in magnetic electron drift mode turbulence is investigated. The mechanism of magnetic Reynolds stress is elucidated with the help of kinetic theory, and a sufficient criterion in the form of Nyquist's criterion for the generation of zonal magnetic fields is developed. This criterion is then applied to a narrow wave packet, where an amplitude threshold due to finite width of the wave spectrum in $k$ space is found. () 2007 American Institute of Physics. [DOI: 10.1063/1.2646436]
\end{abstract}

\section{INTRODUCTION}

The mechanisms for generation of fluid motions with additional symmetry, frequently referred to as flows, have been studied extensively in recent years in electrostatic drift mode turbulence. ${ }^{1}$ In a plasma allowing for inhomogeneities, the gradient-specific modes known as drift-type modes are able to propagate in the direction of translational symmetry, i.e., perpendicularly to the gradient. These modes can then spontaneously generate structures with higher symmetry, the large-scale flows, in a way similar to the established Reynolds stress in hydrodynamics. ${ }^{2}$ The process of generation of such structures is energetically sustained through the wellknown inverse cascade guaranteed in two-dimensional (and quasi-two-dimensional) fluids by the conservation of energy and enstrophy. ${ }^{3,4}$

Among the flows, a limit of great interest is the so-called zonal flows, defined here as symmetric structures with a finite scale in the direction of plasma inhomogeneity, significantly larger than the scale of the underlying small-scale turbulence, and elongated perpendicular to the gradients. ${ }^{4,5}$ This elongation along one defined direction is attributed to the additional symmetry of large-scale flows compared to the small-scale fluctuations.

The generation of such flows in electrostatic drift mode turbulence is of great importance in fusion research, where zonal flows are believed to contribute to enhanced confinement through their shearing effect on the underlying drift waves. ${ }^{6}$ However, zonal flows are not a phenomenon limited to plasma physics. Besides fusion devices, very similar physics is observed in relation with the Rossby waves in both the atmosphere and oceans, where the Rossby waves excite large-scale zonal flows such as the Jet Stream in the atmosphere or the Gulf Stream in the Atlantic ocean.

On the other hand, the excitation of magnetic fields is a current field of strong investigation in different areas. So is the spontaneous generation of strong and large-scale magnetic fields of considerable importance in laser-produced plasmas, which have been observed since the 1970s. The

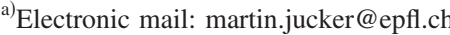

corresponding turbulent fluctuations are drift-type modes excited in a nonuniform unmagnetized plasma, characterized by a frequency range in between the electron and the ion plasma frequencies. Moreover, phenomena occurring in such time scales may even be more important as a source of the secondary magnetic field structures and are often encountered in space physics, ${ }^{7,8}$ as well as in a number of plasmaoperated devices (e.g., switches, focusing devices, Z-pinches, etc.). ${ }^{9}$

It is interesting to combine the phenomena of large-scale structures and strong magnetic fields and thus develop a nonlinear theory capable of describing the generation of such large-scale magnetic fields by small-scale turbulence and their mutual interaction. Such a self-consistent spectral twofield (magnetic field and temperature ${ }^{10}$ ) model is similar to that of electrostatic drift wave turbulence ${ }^{11}$ and has recently been developed in Refs. 12 and 13. Note that this model does not deal with flows in the original sense, since it is not the flow of the particles, but rather magnetic structures that are elongated along one direction and periodical with a long wavelength along the other direction as well. Following this similarity, we call the corresponding large-scale structures "zonal magnetic fields" (ZF), as it has been adopted in the literature. ., $^{1,9,14-17}$

With this nonlinear spectral model, we can focus in the present paper on the detailed generation mechanism of largescale magnetic fields. Two regimes of large-scale magnetic field generation have recently been investigated in Ref. 13 and yielded a general criterion concerning the local form of the wave spectrum in the case of the kinetic regime (where resonance was assumed) and an explicit result for the hydrodynamic regime, where a monochromatic wave spectrum allowed the integration over all modes. In this paper, the goal is to find an instability criterion using a wave kinetic equation similar to the case of kinetic wave theory, where we have the so-called Nyquist criterion. Such a criterion will yield a global statement on whether or not a given equilibrium spectrum can generate zonal magnetic fields. We will follow a similar approach for electrostatic drift waves developed in Refs. 11 and 6. The rest of the paper is organized as 
follows: In Sec. II, we will give a short reminder of the basic equations for magnetic electron drift modes as derived in Ref. 12. In Sec. III, we will follow the derivation of the well-known Nyquist criterion and apply the found general criterion to a narrow wave packet, which can then be compared to the monochromatic case. In Sec. IV, we will conclude the paper with a brief summary.

\section{BASIC EQUATIONS}

The motion of the considered modes is assumed to take place in the plane perpendicular to the magnetic field and hence a quasi-two-dimensional analysis is applied, where only the perturbed magnetic field is directed along the third dimension, here chosen to be the $z$ axis. The considered modes are placed in a nonuniform unmagnetized plasma with density and temperature gradients along the $x$ axis. Magnetic electron drift modes are low-frequency waves with a typical time scale in between the inverse electron and ion plasma frequencies, and consequently the ions play the role of a neutralizing background, whereas the electrons move fast enough to equalize any density perturbation in a relatively short time. Therefore, the electron density will be considered constant in time, $n=n_{0}$. The temperature can be written as the sum of an equilibrium value $T_{0}$ and a perturbation $T$; the perturbed magnetic field is denoted by $B$, having a vanishing equilibrium part.

Starting from the momentum equation and the energy equation, the model equations for magnetic electron drift mode turbulence can be derived and read ${ }^{10}$

$$
\begin{aligned}
& \frac{\partial}{\partial t}\left(B-\lambda^{2} \nabla^{2} B\right)+\beta \frac{\partial T}{\partial y}=\frac{e \lambda^{4}}{m}\left\{B, \nabla^{2} B\right\}, \\
& \frac{\partial T}{\partial t}+\alpha \frac{\partial B}{\partial y}=-\frac{e \lambda^{2}}{m}\{B, T\} .
\end{aligned}
$$

Here $\alpha=\lambda^{2}\left(e T_{0} / m\right)\left(\frac{2}{3} \kappa_{n}-\kappa_{T}\right), \beta=\kappa_{n} / e$. Within the latter definitions, $\kappa_{n}=|\nabla \ln n|$ and $\kappa_{T}=\left|\nabla \ln T_{0}\right|$ are the inverse length scales of the density and the temperature inhomogeneities and $\lambda=c / \omega_{p e}$ is the electron skin depth. The curly brackets on the right-hand side (RHS) denote the Poisson brackets and are defined as $\{a, b\} \equiv(\nabla a \times \nabla b) \cdot \hat{\mathbf{z}}$. The linear dispersion relation can be found from these equations and written as

$$
\omega_{k}=k_{y} \sqrt{\frac{\alpha \beta}{1+k^{2} \lambda^{2}}} .
$$

Note that a purely growing solution is possible for $\kappa_{T}$ $>2 / 3 \kappa_{n}$, which can explain the measured strong magnetic fields in laser-produced plasma experiments. ${ }^{18-20}$ Of course, due to this linear growth, the linear approximation breaks down and nonlinear effects have to be included.

In the nonlinear regime, as has been shown in Refs. 21 and 12, large-scale fields can be generated by small-scale turbulence via magnetic Reynolds stress and a self-consistent theory has been derived describing both large-scale structures and small-scale turbulence using a wave kinetic equation for an appropriate action-like invariant, taking the form

$$
\left|N_{k}\right|=\left|\psi_{k}\right|^{2}=4 \frac{\alpha}{\beta}\left(1+k^{2} \lambda^{2}\right)\left|B_{k}\right|^{2} .
$$

The corresponding wave kinetic equation has been shown to be $^{12}$

$$
\frac{\partial N_{k}}{\partial t}+\frac{\partial \omega_{k}^{\mathrm{NL}}}{\partial \mathbf{k}} \frac{\partial N_{k}}{\partial \mathbf{r}}-\frac{\partial \omega_{k}^{\mathrm{NL}}}{\partial \mathbf{r}} \frac{\partial N_{k}}{\partial \mathbf{k}}=2 \gamma_{k} N_{k}-\operatorname{St}\left(N_{k}\right),
$$

where $\omega_{k}^{\mathrm{NL}}$ is the Doppler-shifted linear frequency $\omega_{k}$ due to the presence of large-scale magnetic fields. The terms on the RHS account for wave damping due to linear and nonlinear mechanisms, as well as local wave interactions, and guarantee saturation in the absence of large-scale fields.

In the next section, we will elaborate a criterion in the form of the well-known Nyquist criterion, which will give a global condition to be fulfilled by the wave spectrum for large-scale magnetic field generation.

\section{NYQUIST'S CRITERION}

We will use Fourier decomposition and scale separation in order to express zonal magnetic fields on the one hand and small-scale turbulence on the other hand. Explicitly, we write the two parts of the total wave spectrum as $(B, T)(t)$ $=\Sigma_{k}\left(B_{k}, T_{k}\right)(t) e^{i \mathbf{k r}}+\left(B_{q}, T_{q}\right)(t) e^{i \mathbf{q r}}$, where $|\mathbf{q}| \ll|\mathbf{k}|$. Then, applying the condition for zonal magnetic fields, $\mathbf{q}=(q, 0,0)$, the model equations (1) yield the evolution equations for the large-scale magnetic field and temperature, ${ }^{12,21}$

$$
\begin{aligned}
& \frac{\partial B_{q}}{\partial t}=\frac{e \lambda^{2}}{m} \frac{q^{2} \lambda^{2}}{1+q^{2} \lambda^{2}} \int\left|B_{k}\right|^{2} k_{x} k_{y} d^{2} \mathbf{k}, \\
& \frac{\partial T_{q}}{\partial t}=0,
\end{aligned}
$$

where the sum over the harmonics has been replaced by an integral, and we neglected the noise emitted into the fields by incoherent coupling of the magnetic electron drift modes. It is a direct consequence from these equations that the mean electron temperature associated with zonal magnetic fields does not evolve with time. The square of the magnetic Fourier amplitude in (5a) can be replaced by the wave spectrum using its definition (3). Furthermore, with the decomposition of the wave spectrum $N_{k}=N_{k}^{0}+\tilde{N}_{k}$, the equilibrium distribution $N_{k}^{0}$ has to satisfy

$$
\int \frac{N_{k}^{0}}{1+k^{2} \lambda^{2}} k_{x} k_{y} d^{2} \mathbf{k}=0,
$$

which can easily be seen from (5a). Further Fourier expansion of the large-scale magnetic field in time, $B \sim B_{q} \exp [$ $-i \Omega t]$, yields from (5) and (3) the dispersion relation

$$
\Omega=K_{q}^{2} q^{2} \int d^{2} \mathbf{k} \frac{k_{y}^{2} \lambda^{2}}{1+k^{2} \lambda^{2}} k_{x} \frac{\partial N_{0}}{\partial k_{x}} \frac{1}{\Omega-q v_{g}} .
$$

For the group velocity $v_{g}$, only the linear frequency is considered, since the nonlinear contribution gives rise to a Doppler shift that is not of interest here. Thus, the group velocity is 


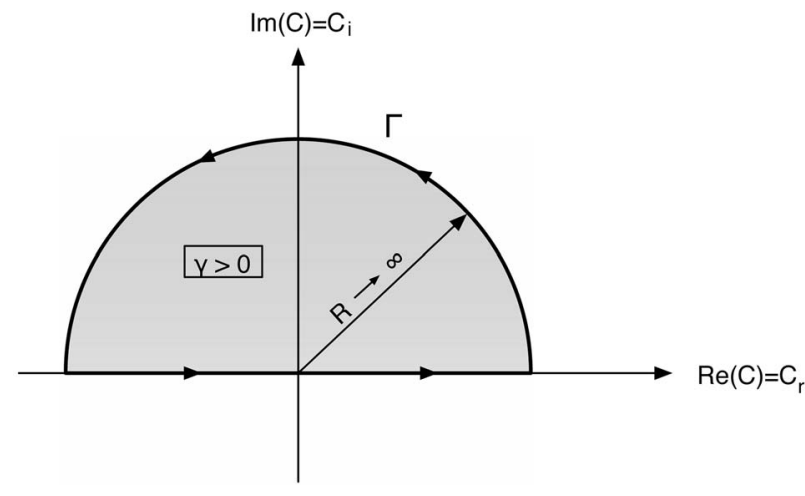

FIG. 1. The integration path $\Gamma$ in complex phase velocity space.

$$
v_{g}=\frac{\partial \omega_{k}}{\partial k_{x}}=-\sqrt{\alpha \beta} \frac{k_{x} k_{y} \lambda^{2}}{\left(1+k^{2} \lambda^{2}\right)^{3 / 2}} .
$$

Note that the group velocity is symmetric with respect to the wave-vector components $k_{x}$ and $k_{y}$. As a next step, we express the $x$ component of the wave vector as a function of $v_{g}$ and assume the group velocity in resonance with the phase velocity of the zonal field perturbation, $v_{g} \approx \Omega / q$, so that (7) can be written

$$
1+\frac{K_{q}^{2} q}{\sqrt{\alpha \beta}} \int \frac{k_{y} d^{2} \mathbf{k}}{\Omega-q v_{g}} \frac{\partial N_{0}}{\partial k_{x}} \sqrt{1+k^{2} \lambda^{2}}=0 .
$$

We want to change variables $\left(k_{x}, k_{y}\right) \rightarrow\left(V, k_{y}\right)$, where $V$ is the group velocity $V=V(\mathbf{k})$. For the integration limits, the extrema of $v_{g}$ have to be found. Deriving the group velocity (8) yields that at the extrema, $\left|v_{g}^{m}\right|=\sqrt{\alpha \beta / 27}$ and $k^{2} \lambda^{2}=2$. So, at the integration boundaries, $k \lambda \approx 1$. But for $k \lambda>1, v_{g} \approx 0$ most of the time. Therefore, we restrict ourselves to the limit $k \lambda \ll 1$ only. Furthermore, let us define the one-dimensional wave spectrum $K(V) \equiv \int_{V=c s t} N_{0} k_{y} d k_{y}$ and the phase velocity $C \equiv \Omega / q$, so that the dispersion relation now reads

$$
\Lambda(C) \equiv 1-\frac{K_{q}^{2}}{\sqrt{\alpha \beta}} \int_{-V_{0}}^{V_{0}} \frac{K^{\prime}(V) d V}{V-C}=0,
$$

where $V_{0} \equiv \max \left|v_{g}\right|=\sqrt{\alpha \beta / 27}$. The latter equation has exactly the form of the dispersion relation in wave kinetic theory of plane waves. ${ }^{6}$ We will thus treat the integral in (10) as a Landau integral in a complex plane. The distribution $K(V)$ is now given as a function of the perturbation group velocity $V$, which is in resonance with the zonal field perturbation phase velocity $C$.

In order to have an instability, we need by definition of $C$ an imaginary part of the phase velocity, $C=C_{r}+i C_{i}$, with $C_{i}>0$, because in that case $\Omega=\Omega_{r}+i \gamma$ with $\gamma=q C_{i}>0$. We follow the standard derivation of the Nyquist criterion and consider $\Lambda(C)$ as a mapping from $C$ to $\Lambda$ space. Instabilities occur for $C_{i}>0$. Hence, we consider the path $\Gamma$ in $C$ space along the real axis at $C_{i}=0^{+}$and with an infinite radius in the upper half of $C$ space (Fig. 1).

If at a point in $\operatorname{int}(\Gamma), \Lambda(C)=0$, there is an instability since there is a solution of the dispersion relation with $\gamma$ $>0$. Let us note that within int $(\Gamma), \Lambda$ has no singularities, since $C_{i}>0$ and $V \in \mathbb{R}$, so that $V-C \neq 0$. Then, a zero in

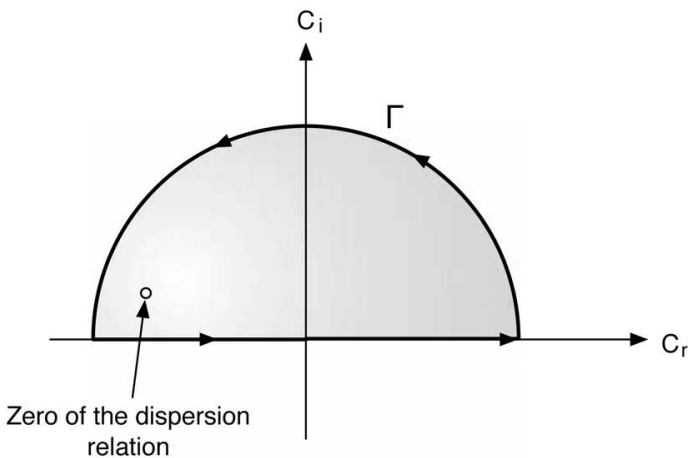

(a)

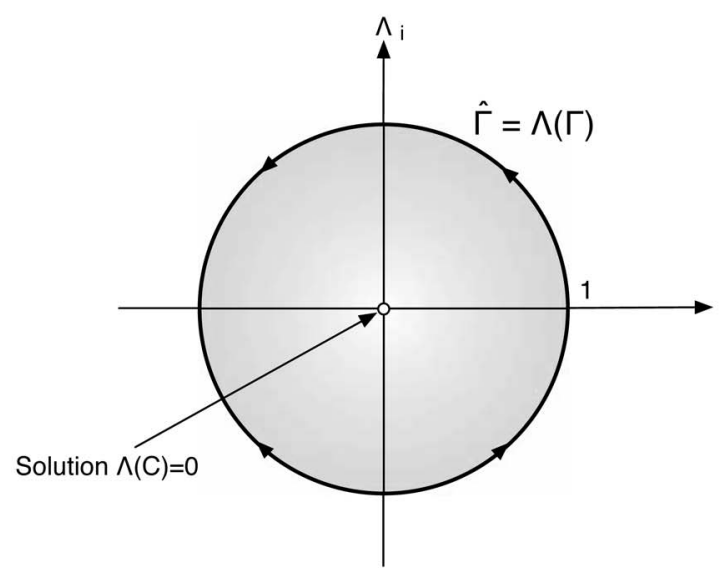

(b)

FIG. 2. The number of zeros in $\operatorname{int}(\Gamma)$ corresponds to the number of rotations around the origin in $\Lambda$ space. Note that $\Lambda \rightarrow 1$ for $C \rightarrow \pm \infty$.

$\operatorname{int}(\Gamma)$ corresponds to circling a solution of the dispersion relation counterclockwise. For the mapping $\Lambda(C)$ this means rotating around the origin counterclockwise (Fig. 2), i.e., that the number of zeros in $\operatorname{int}(\Gamma)$ corresponds to the number of rotations around $\Lambda=0$ in $\Lambda$ space. Thus, we conclude that if we have no rotations around the origin in $\Lambda$ space, there is no solution of the dispersion relation with positive increment and therefore no instability, i.e., the system is stable. But for a rotation around the origin, the path $\hat{\Gamma} \equiv \Lambda(\Gamma)$ must cross the positive real axis from below, which corresponds to $\Lambda_{i}$ changing sign from negative to positive at $\Lambda_{r}>0$, and can be expressed mathematically as $\Lambda_{i}=0$ and $\Lambda_{i}^{\prime}>0$ at $\Lambda_{r}>0$ for a possible instability. But treating the dispersion relation (10) as a Landau integral, we know that at the real axis

$$
\begin{aligned}
\Lambda\left(C_{r}\right)= & 1-\frac{K_{q}^{2}}{\sqrt{\alpha \beta}} \mathrm{P} \cdot \mathrm{V} \cdot \int_{-V_{0}}^{V_{0}} \frac{K^{\prime}(V) d V}{V-C_{r}} \\
& -\left.i \pi \frac{K_{q}^{2}}{\sqrt{\alpha \beta}} K^{\prime}(V)\right|_{V=C=C_{r}} .
\end{aligned}
$$

It can easily be seen from this that $\Lambda_{i}=0 \Leftrightarrow K^{\prime}\left(C_{r}\right)=0$ and $\Lambda_{i}^{\prime}>0 \Leftrightarrow K^{\prime \prime}\left(C_{r}\right)<0$, i.e., a possible instability occurs at a maximum of $K(V)$. So, if at every point on the positive real axis in $\Lambda$ space, the condition $\Lambda_{i}^{\prime}<0$ is satisfied, there is no instability since there is no rotation around the origin. In terms of the distribution function $K(V)$, this means that if at 
every minimum of $K(V), \Lambda$ is positive, the distribution is stable, or, more mathematically,

$$
\begin{aligned}
& \text { if } \forall C_{r} \text { with } K^{\prime}\left(C_{r}\right)=0, K^{\prime \prime}\left(C_{r}\right)>0 \text {; } \\
& \frac{K_{q}^{2}}{\sqrt{\alpha \beta}} \int_{-V_{0}}^{V_{0}} \frac{K^{\prime}(V) d V}{V-C_{r}}<1,
\end{aligned}
$$

then the distribution is stable. But we are more interested in an instability than a stability criterion. Remember the definition of $K(V)$ as a function of the two-dimensional equilibrium distribution $N_{0}$. Furthermore, as already mentioned, $N_{0}$ must satisfy Eq. (6). From the latter equation, we get the idea to assume that $N_{0}$ is symmetric in $V$ and $k_{y}$, i.e., $N_{0}\left(V, k_{y}\right)$ $=N_{0}\left(V,-k_{y}\right)=N_{0}\left(-V, k_{y}\right)$. Then, $K(V)=K(-V)$ and $K^{\prime}(V)=$ $-K^{\prime}(-V)$. It then follows from the expression (11) that $\Lambda\left(C_{r}\right)=\Lambda\left(-C_{r}\right)^{*}$ and thus $\hat{\Gamma}\left(C_{r}<0\right)=\hat{\Gamma}\left(C_{r}>0\right)^{*}$, i.e., if $\hat{\Gamma}$ crosses the real axis at $\Lambda_{r}<0$, which is necessary for rotation around the origin, i.e., instability, it can recross at $\Lambda_{r}<0$ (and thus no instability) only if $K(V)$ has more than five extrema (Fig. 3). Therefore, we can formulate a sufficient criterion for instability: If $K(V)=K(-V)$ and $K(V)$ has one or three extrema and if

$$
\frac{K_{q}^{2}}{\sqrt{\alpha \beta}} \int_{-V_{0}}^{V_{0}} \frac{K^{\prime}(V) d V}{V-C_{r}}>1
$$

for at least one extremum $V=C_{r}$, then the distribution is unstable. It is then obvious that the extremum has to be a minimum (the integral has to be positive), and the slopes around the minimum have to be steep enough for the integral to be greater than 1 .

Another way of writing the same criterion is to use the symmetry conditions for $K(V)$ to get the following statement: If the symmetric function $K(V)$ has fewer than five extrema in the interval $\left(-V_{0}, V_{0}\right)$ and at least one point $V=C_{r}$ where $K^{\prime}\left(C_{r}\right)=0$, then

$$
\frac{2 K_{q}^{2}}{\sqrt{\alpha \beta}} \int_{0}^{V_{0}} \frac{V K^{\prime}(V) d V}{V^{2}-C_{r}^{2}}>1
$$

guarantees instability. As an example, we will use this criterion for a narrow wave packet, which is the simplest distribution for the wave spectrum. Another reason for this example is its similarity with the case of a monochromatic distribution, which was investigated earlier in Ref. 13.

\section{A. Narrow wave packet}

For a narrow wave packet, the mean distribution function can be written

$$
N_{0}(\mathbf{k})=\frac{N_{0}}{2 \pi \Delta k^{2}}\left(e^{-\left(\left|\mathbf{k}-\mathbf{k}_{0}\right|^{2} / \Delta k^{2}\right)}+e^{-\left(\left|\mathbf{k}-\mathbf{k}_{1}\right|^{2} / \Delta k^{2}\right)}\right) .
$$

In order to satisfy the equilibrium condition (6), we choose $k_{0 x}=-k_{1 x}$ and $k_{0 y}=k_{1 y}$. The condition for a narrow wave packet is $\Delta k \ll k_{0}$. Then, we can expand $V(\mathbf{k})$ around $\mathbf{k}=\mathbf{k}_{0}$,

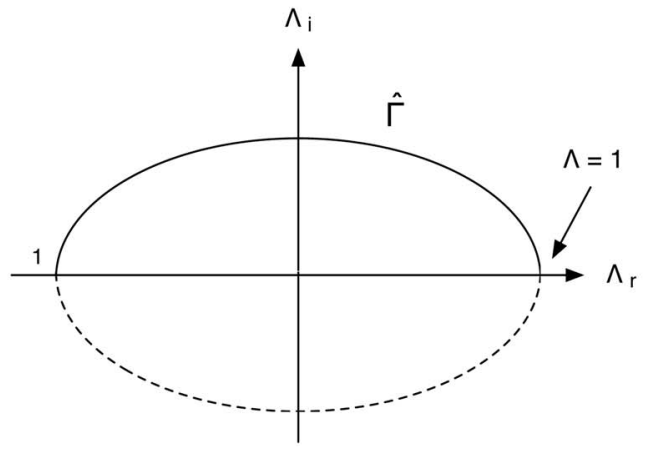

(a)

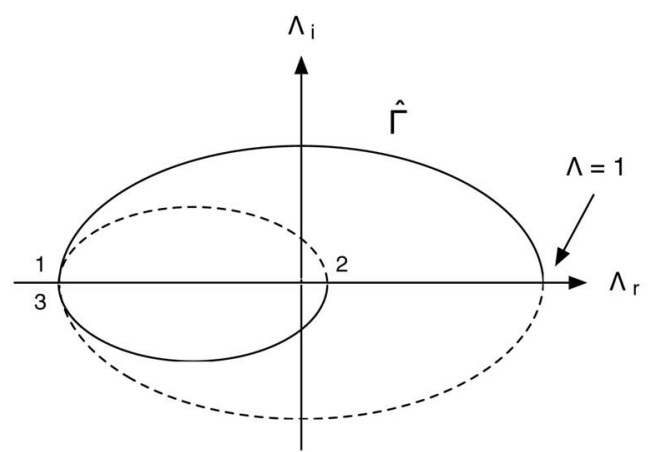

(b)

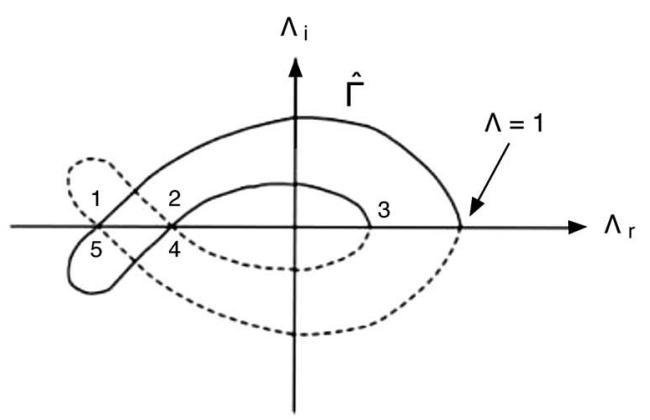

(c)

FIG. 3. Examples of distributions with five, three, or one extremum. A distribution crossing the real axis at $\Lambda_{r}<0$ but without instability is only possible with five or more extrema. The solid lines denote $\hat{\Gamma}\left(C_{r}<0\right)$, the dotted lines $\hat{\Gamma}\left(C_{r}>0\right)=\hat{\Gamma}\left(C_{r}<0\right)^{*}$.

$$
V(\mathbf{k}) \cong V\left(\mathbf{k}_{0}\right)+\left.\frac{\partial V}{\partial \mathbf{k}}\right|_{\mathbf{k}_{0}} \cdot\left(\mathbf{k}-\mathbf{k}_{0}\right) .
$$

Note that it is assumed that $\Delta k \ll\left|\mathbf{k}_{0}-\mathbf{k}^{*}\right|$, where the derivative of $V$ is zero at $\mathbf{k}=\mathbf{k}^{*}$. If this is not the case, one has to include the second-order term of the expansion. Let us denote $V_{k_{0}} \equiv V\left(\mathbf{k}_{0}\right)$ and $V_{x, y} \equiv \partial V / \partial k_{x, y}$. Then, we express $k_{x}$ as a function of $V$ and $k_{y}$ through (16), put the distribution into the definition of $K(V)$, and use the narrow wave-packet condition as well as $k_{x}, k_{y}$ symmetry, such that the derivative reads

$$
K^{\prime}(V) \cong-\frac{2 V_{x}}{\left(V_{x}^{2}+V_{y}^{2}\right)^{3 / 2}} \frac{N_{0} k_{0 y}}{\sqrt{\pi} \Delta k^{3}} e^{-\left[\left(V-V_{k_{0}}\right)^{2} /\left(V_{x}^{2}+V_{y}^{2}\right) \Delta k^{2}\right]} .
$$

The minimum of the distribution is at $V=V_{k_{0}}$, so that $C_{r}$ $=V_{k_{0}}$ in the instability criterion (14). In order to calculate the 
integral in Nyquist's criterion, we neglect corrections of the second order and remember that the calculations are only valid in the limit $k \lambda \ll 1$ together with the fact that $k \lambda=\sqrt{2}$ at $V=V_{0}$, so that the upper limit of the integral can be treated infinite. The resulting inequality is then

$$
-\frac{N_{0} k_{0 y} K_{q}^{2}}{\sqrt{\alpha \beta} \Delta k^{2}} \frac{V_{x}}{V_{x}^{2}+V_{y}^{2}}>1 .
$$

As a first result, we find a criterion similar to the Lighthill criterion in the case of modulational instability, $v_{g}^{\prime}<0$, for instability. Explicit calculations yield finally

$$
\frac{N_{0} K_{q}^{2}}{\alpha \beta \Delta k^{2}}\left(\frac{k_{0 y}}{k_{0}}\right)^{2}>1
$$

This result has the feature that the stability depends on the relative strength of the components of the wave packet center $\mathbf{k}_{0}$. If $k_{0 y}$ is too small compared to $k_{0}$, the distribution is stable. This is the same statement as found in the monochromatic case. ${ }^{13}$ But in addition to this statement, we find out here that due to finite width $\Delta k$, there is a threshold for the amplitude of the order of $N_{0} \sim \alpha \beta \Delta k^{2} / K_{q}^{2}$, which is proportional to the squares of both the inverse characteristic inhomogeneity lengths and the width of the wave packet.

\section{CONCLUSIONS}

In this paper, the properties of magnetic electron drift wave turbulence are studied and a criterion for generation of zonal magnetic fields by small-scale turbulence is investigated. Based on similarities to wave kinetic theory, we have shown that an instability criterion including the global properties of the wave spectrum similar to Nyquist's criterion can be found and calculated in its explicit form.

\section{ACKNOWLEDGMENTS}

One of the authors (M.J.) would like to acknowledge the hospitality of the Department of Astronomy and Space Physics during his stay at Uppsala University, as well as the support and open-mindedness of Professor Laurent Villard and the École Polytechnique Fédérale in Lausanne, Switzerland, in the frame of bilateral scientific exchange.

${ }^{1}$ P. H. Diamond, S.-I. Itoh, K. Itoh, and T. Hahm, Plasma Phys. Controlled Fusion 47, 35 (2005).

${ }^{2}$ P. Diamond and Y.-B. Kim, Phys. Fluids B 3, 1626 (1991).

${ }^{3}$ J. Charney, J. Atmos. Sci. 28, 1087 (1971).

${ }^{4}$ A. Hasegawa, C. Maclennan, and Y. Kodama, Phys. Fluids 22, 2122 (1979).

${ }^{5}$ F. Busse, Chaos 4, 123 (1994).

${ }^{6}$ M. A. Malkov, P. H. Diamond, and A. Smolyakov, Phys. Plasmas 8, 1553 (2001).

${ }^{7}$ A. Smolyakov, P. Diamond, and Y. Kishimoto, Phys. Plasmas 9, 3826 (2002).

${ }^{8}$ W. P. Wilkinson, Planet. Space Sci. 51, 629 (2003).

${ }^{9}$ A. Das and P. H. Diamond, Phys. Plasmas 7, 170 (2000).

${ }^{10}$ J. Nycander, V. Pavlenko, and L. Stenflo, Phys. Fluids 30, 1367 (1987).

${ }^{11}$ M. A. Malkov and P. H. Diamond, Phys. Plasmas 8, 3996 (2001).

${ }^{12}$ Zh. N. Andrushchenko, M. Jucker, and V. P. Pavlenko, "Self-consistent model of electron drift mode turbulence," J. Plasma Phys. (to be published).

${ }^{13}$ M. Jucker, Zh. N. Andrushchenko, and V. P. Pavlenko, Phys. Plasmas 13, 072308 (2006)

${ }^{14}$ A. Das, R. Singh, P. Kaw, and S. Champeaux, Phys. Plasmas 9, 2609 (2002).

${ }^{15}$ C. Holland and P. Diamond, Phys. Plasmas 9, 3857 (2002).

${ }^{16}$ K. Subramanian, Phys. Rev. Lett. 90, 245003 (2003).

${ }^{17}$ L. Rudakov, Phys. Plasmas 12, 042111 (2005).

${ }^{18}$ M. Tatarakis et al., Phys. Plasmas 9, 2244 (2002).

${ }^{19}$ S. Q. Liu and X. Q. Li, Phys. Plasmas 7, 3405 (2000).

${ }^{20}$ M. Y. Yu, P. K. Shukla, and L. Stenflo, Phys. Fluids 30, 10 (1987).

${ }^{21}$ Zh. N. Andrushchenko and V. P. Pavlenko, Phys. Plasmas 11, 1402 (2004). 